\title{
Cortical Brain Functions - The Brodmann Legacy in the 21st Century
}

\section{Funções corticais cerebrais - O legado de Brodmann no século XXI}

\author{
Daniel Damiani $^{1,2}$ Anna Maria Nascimento ${ }^{1}$ Leticia Kühl Pereira ${ }^{1}$ \\ ${ }^{1}$ Department of Neuroscience, Universidade Anhembi Morumbi, São \\ Paulo, SP, Brazil \\ 2 Department of Neurology, Hospital do Servidor Público do Estado \\ (IAMSPE/HSPE), São Paulo, SP, Brazil \\ Address for correspondence Daniel Damiani, MD, Universidade \\ Anhembi Morumbi, São Paulo, SP, Brazil \\ (e-mail: damiani.neuroscience@gmail.com).
}

Arq Bras Neurocir 2020;39(4):261-270.
Abstract
Keywords
- Brodmann areas
- brain cortical areas
- intelligence circuitry
- brain functions
- new brain areas
- learning circuitry

\section{Resumo}

Palavras-chave

- áreas de Brodmann

- áreas corticais cerebrais

- circuito da inteligência

- funções cerebrais

- novas áreas cerebrais

- circuito do aprendizado
In 1909, Korbinian Brodmann described 52 functional brain areas, 43 of them found in the human brain. More than a century later, his devoted functional map was incremented by Glasser et al in 2016, using functional nuclear magnetic resonance imaging techniques to propose the existence of 180 functional areas in each hemisphere, based on their cortical thickness, degree of myelination (cortical myelin content), neuronal interconnection, topographic organization, multitask answers, and assessment in their resting state. This opens a huge possibility, through functional neuroanatomy, to understand a little more about normal brain function and its functional impairment in the presence of a disease.

Em 1909, Korbinian Brodmann descreveu 52 áreas funcionais cerebrais, sendo 43 delas encontradas no cérebro humano. Seu consagrado mapa funcional foi incrementado por Glasser et al em 2016, utilizando técnicas de ressonância nuclear magnética funcional para propor a existência de 180 áreas funcionais em cada hemisfério, baseadas em sua espessura cortical, grau de mielinização, interconexão neuronal, organização topográfica, respostas a multitarefas ,e avaliação no seu estado de repouso. Abre-se uma enorme possibilidade, por meio da neuroanatomia funcional, de se entender um pouco mais sobre o funcionamento cerebral normal e do seu comprometimento funcional na presença de uma doença.

\section{Introduction}

Korbinian Brodmann ${ }^{30}$ became a milestone for modern neuroscience with the publication of his monograph in 1909. The path toward the finding of cerebral cortical functions was initiated there. With technological advancement, the neuroimaging techniques currently available have transformed the knowledge about the human brain. Positron emission tomography (PET), functional nuclear magnetic resonance (fNMR), electroencephalography (EEG), electrocorticography (ECoG), magnetoencephalography (MEG), and, more recently, optical infrared spectroscopy (OIRS) have been opening the field for the discovery of cerebral cortical functions, enabling a greater understanding of brain diseases, and increasing, as never before, neurophysiological knowledge. Associated with macroscopic knowledge provided by neuroimaging, microscopic knowledge has also made contributions: optogenetics and molecular biology are fundamental tools to understand brain received

August 11, 2016

accepted

November 9, 2016
DOI https://doi.org/

10.1055/s-0036-1597573. ISSN 0103-5355.
Copyright $(2020$ by Thieme Revinter

Publicações Ltda, Rio de Janeiro, Brazil
License terms

(c) $(1) \$$ 
functioning. Peng et $\mathrm{al}^{6}$ published several studies confirming the functional division proposed through the gene signature present in each anatomical region to be plausible. More than a century after the publication of Brodmann, Glasser et al published, in $2016^{2}$, a new functional cortical map. Based on several fNMR studies present in the Human Connectome Project (HCP) databases, the degrees of cortical myelinization and cortical thickness were observed by MRI images weighted in high resolutions $\mathrm{T} 1$ and $\mathrm{T} 2$, while the cortical function was checked by blood oxygen level dependent (BOLD) fNMR during multitask tests. The connectivity and topography were also evaluated by fNMR in the cerebral rest states. Glasser et $\mathrm{al}^{2-4}$ described 180 functional areas present in each cerebral hemisphere, promising to add to the original Brodmann ${ }^{30}$ map the information currently available. No technology alone will offer the answers that the neuroscientific community seeks, but the various methods used together, associated with those already established, such as the functional map of Brodmann, ${ }^{30}$ will provide this functional understanding of the brain..$^{1-6}$

\section{Objective}

The authors present the result of several studies demonstrating the current knowledge about cerebral cortical functions and correlate the current knowledge obtained through functional neuroimages with those recommended by Korbinian Brodmann ${ }^{30}$ more than a century ago.

\section{Materials and Methods}

A quantitative and descriptive research through a narrative review of the literature was conducted in the Latin American and Caribbean Health Sciences Literature (LILACS, in Portuguese) and the National Library of Medicine (PubMed) databases in August 2016, using the following descriptors: Brodmann areas; brain cortical areas; intelligence circuitry; brain functions; new brain areas; and learning brain circuitry.

The present study has the following guiding question: what do we currently know about cerebral cortical functions that is still related to those proposed by Brodmann in 1909? The inclusion criteria were: updated publications from 2001 to 2016, with rare exceptions, written in Portuguese, Spanish and English, with online access to the full text. Duplicate articles were excluded. For the analysis of the articles included in this review, the following aspects were observed: year of publication, type of periodical, place where the study was conducted, methodology used, and main results.

\section{Development}

Historical aspects of the functional cortical mapping. In 1909, Korbinian Brodmann, ${ }^{30}$ a German physician, published a monograph with the experiments he performed in the laboratories of Oskar and Cécilie Vogt in Berlin. Influenced by the ideas of phrenologists Gall and Spurzheim, Brodmann integrated the cytoarchitecture found by Vogt et al and the functional information published by Wernicke and Broca.
Through the observations that Brodmann ${ }^{30}$ found in the gray matter of the brain, considering the presentation of the number of cell layers, cellular morphology, distribution of dendritic connections and neuronal types, the cortical areas were divided into numbers, a division that is still used today in modern neuroscience. Brodmann used animal models to create a numerical homology corresponding to the cortical areas; he found 43 functional areas ( 11 regions) attributed to the human cerebral cortex, and, in other primates, 52 areas are described. The areas are numbered between 1 and 52, with no homology between areas 12 and 16 and 48 and 51, which are present in monkeys. Moreover, in his classic monograph, Brodmann ${ }^{30}$ points out that the cellular polymorphism and the connective network in the human cerebral cortex have much higher complexity when compared with those of other primates ( - Fig. 1). Finally, in 1909, Brodmann published his monograph, ${ }^{30}$ in which only 36 of the 324 pages are devoted to the cortical functional reports found in the human brain. However, 5 years later, Brodmann ${ }^{8}$ published an extensive study describing the functional cytoarchitectural areas of the brain with electrophysiological correlations observed in animals undergoing brain injuries. ${ }^{7,8}$ Neuroscience has advanced considerably in the last century after its publication. Great discoveries in the fields of clinical neurology, neurosurgery and psychiatry were based on the functional map described by Brodmann. In addition, Vogt et al used studies with myelination patterns to subdivide the Brodmann areas into subareas, highlighting intracortical regional fibers, thus creating a combination of Brodmann's cytoarchitectural areas with their functional myeloarchitectonic maps. In 1925, Von Economo and Koskinas published a detailed cytoarchitectural description of the cortical areas, adding to it information not mentioned by Brodmann. Subsequent maps, including those of the Russian School (1949), only pay tribute to the concepts of Brodmann, changing the extensions of the previously described areas. Lashley and Clark highlighted that, despite the efforts by Brodmann $^{8,30}$ and Vogt, their works are difficult to reproduce due to the great individual variability of cortical structures, which is even more pronounced when other animal species are considered. ${ }^{7}$

Applied Neurotechnology. In the 1980s, once again the concepts of Brodmann gained prominence in the neurosciences. With the introduction of fNMR techniques, it became possible to observe a living brain in full neurophysiological activity. Through several study protocols, the various tasks demanded by neurons were verified by resonance images, offering the possibility of a real functional cortical mapping. Brodmann's functional maps were quickly recreated three-dimensionally through software, with new conceptions of extremely precise and individualized stereotaxic maps. The intrasulcal regions, which are responsible for two thirds of the total cortical surface, and were not considered by Brodmann, were remapped, giving rise to several stereotactic atlases currently used (such as the Atlas of Talairach and Tournoux, for example). In the 21st century, technological tools offer multimodal maps, not only regarding cytoarchitectural information, but correlating it with cerebral neurocircuitry, molecular organization, genetics and 

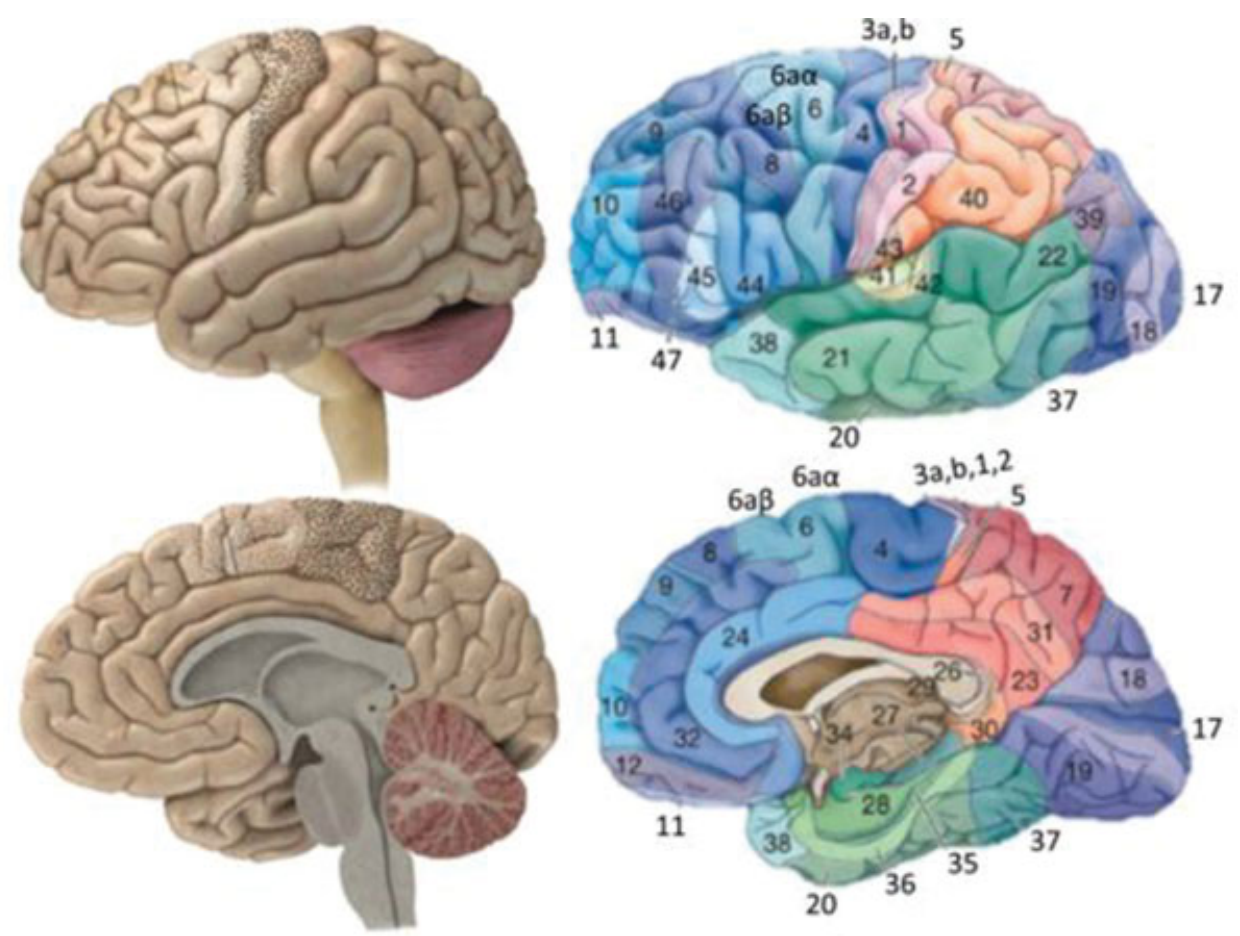

Fig. 1 Traditional functional areas described in 1909 by Korbinian Brodmann, 43 in the human species, 8 with functional characterizations implemented with neuroimaging exams. The black dotted line represents the primary motor cortex or AB4, and the white dotted line, the premotor and supplementary motor areas, which together are called AB6.

individual functional aspects. The NMR by diffusion tensor imaging (DTI) reveals the correlated subcortical structures, emphasizing neurocircuitry with the visualization of the white fibers that interconnect multiple cortical and subcortical centers. ${ }^{9-11}$

Brodmann's functional areas - classic concepts updated by fNMR. Out of the 43 functional areas (BA [Brodmann Area]) Brodmann described in his cytoarchitectural study, ${ }^{8}$ some have greater clinical relevance ( - Fig. 1). AB3a/b,1,2 (primary sensory areas): located in the postcentral gyrus, therefore, posterior to the central groove, in the territory of the parietal lobe, they receive afferences from the thalamus, with primary sensory information. They receive nociceptive, thermal information from the protopathic (coarse) touch, the epicritic touch (fine), vibration, and conscious proprioception. Neurons in area 3 a receive input signals from neuromuscular spindles and other deep receptors. Neurons present in area $3 \mathrm{~b}$ receive signs of specific classes of touch receptors in the skin. Neurons in areas 1 and 2 receive convergent input signals from multiple types of somatosensory receptors that innervate the same parts of the body. The clinical significance of the lesions present in the postcentral gyrus may correspond to paresthesia, anesthesia, hypoesthesia (thermal, tactile or painful). ${ }^{12,13}$ The involvement of secondary and tertiary areas of sensitivity corresponding to AB 5 and 7 (upper parietal lobe), area 5 neurons are active mainly in the active movements of the hand and in the modalities of integrated sensitivity (protopathic and epicritic), and its lesions can be clinically studied with: apraxia (ideomotor, ideational, constructive and dressing apraxia), dysgraphia, hemineglect, graphesthesia, stereognosis and spatial disorientation. Anatomically, the intraparietal sulcus divides the inferi- or upper parietal lobe, creating a band around the intraparietal sulcus called the V2 area, which, along with the AB3a area, receives information from the vestibular system, with important control of body balance. AB4 (primary motor area): located in the precentral gyrus, anterior to the central groove, it has the pyramidal cells of Betz, thus originating the corticospinal and corticonuclear tracts, which are responsible for the initiation of the voluntary motor act. A lesion in this structure will be represented by paresis, and rarely plegia due to the complementation of the motor act by secondary pathways, which are also called extrapyramidal. The motor area of the speech, named by Brodmann as AB44 (also called the Broca area) is very close to the precentral gyrus, being responsible for the speech motor act (voluntary). The premotor cortex and supplementary motor area are immediately anterior to the precentral gyrus, in the frontal lobe, and correspond to the

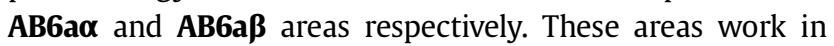
combination with the precentral gyrus in motor programming. Recent studies on neuroimaging reveal that the supplementary motor area is responsible for motor memory, that is, it stores information about motor acts already learned, while the premotor area uses its cells to learn new motor schemes. Lesions in these structures are related to ideomotor apraxia, loss of motor sequence of movements, including walking and the harmonious sequence of movement. If the lesion is large enough to involve the Broca area, motor aphasia or motor transcortical aphasia may be perceived. The control area of the contralateral conjugated gaze (horizontal balcony movements), or $\mathbf{A B 8}$, is located anteriorly to AB6. The lesions in this area classically course with deviation from the conjugated gaze to the side of the lesion (déviation conjuguée); however, in 
addition to this function, the neuroimaging revealed that this region also participates in executive functions (planning, reasoning, learning engine and behavior), language and working memory. Some authors report that lesions in this area may be related to mutism and/or difficulty to initiate speech. The prefrontal area, which is uniformly related to the control of behavior, is located more anteriorly to AB8. AB9 and $\mathbf{1 0}$ are called the dorsolateral prefrontal cortex. They occupy the largest portion of the middle frontal gyrus, which is responsible for working memory, that is, for intellectual functions, memory, focus, decision-making and attention. The lesions in these structures will heal with dysexecutive syndromes, causing difficulty in reasoning, concentration, decision-making and abstract thinking. The mechanisms of consolidation of memories, as well as the processes of voluntary forgetfulness, depend on the functioning of this region, which is compromised in the presence of injuries. The medial portions of these regions (AB9 and 10) correspond to the ventromedial prefrontal cortex, which is related to the emotional aspects of the aforementioned executive functions, including meditation, judgment, reward circuit and benefit versus disadvantage in decision-making or choice. Still in the prefrontal cortex, there is a third anatomo-functional subdivision called AB11,12, or orbitofrontal cortex. This region is associated with attention, focus, hyperactivity and behavior (impulsiveness). Injuries in this region are related to loss of social censorship, hypersexuality, impairment in social interaction, drug abuse and obsessive-compulsive behavior. The occipital lobe holds the circuits related to vision, consisting of several areas: $\mathbf{A B 1 7}$ or $\mathbf{V 1}$, which is considered the primary visual cortex; the upper visual field is projected beneath the calcarine groove, while the inferior visual field is projected above the calcarine sulcus of the occipital lobe. The lesions in V1 regions course with homonymous hemianopsia, heteronomous hemianopsia, quadrantanopsia, scotomas, complete blindness or bilateral amaurosis. AB17 identifies information corresponding to luminosity, shape, color, pattern, and movement of objects. However, AB18 or V2, which is classified as the secondary or prestriated visual cortex, is responsible for the first visual associative information. The V2 area processes complex information about the shape and contour of objects, and the layer of neurons present in V2 is responsible for the visual memory and its long-term consolidation, in addition to recognizing objects. The clinical expression of lesions in V2 may course with visual agnosia, achromatopsia, prosopagnosia and topographic agnosia. Lesions in V2 on the left classically course with alexia. It is noteworthy that studies with fNMR show that the V2 area often works with AB19 and 37. Finally, AB19 (associative visual cortex, which is also part of the Wernicke area), or areas $\mathbf{V} \mathbf{3}$ and $\mathbf{V} \mathbf{5}$ (dorsal), shares the same functions as AB18; However, some functions such as precise spatial location, and telling the individual where the visualized object is in space, belong exclusively to AB19. Still belonging to AB19, the V4 area (ventral) corresponds to the interpretation of the meaning of the object, telling the individual what is being seen. With functional neuroimaging techniques, there are more and more retinotoxic areas subdividing AB19, but it is not the purpose of the present article to highlight them. Some psychiatric syndromes are manifested by alterations in these regions, such as Capgras syndrome and Fregoli syndrome. ${ }^{14-16}$ Present in the lower temporal gyrus, in lateral view, AB20 is responsible for functions related to the recognition of objects and, consequently, is part of the memory mechanisms. Studies with fNMR recently revealed that $A B 20$ is part of the complex of regions related to language comprehension, being also part of the Wernicke area. AB20 relates to lexical and semantic processing, understanding of metaphors, and production and interpretation of language. In parallel, also in this region, there is participation of visual processing, including the perception of objects in a scenario. Due to the proximity of the structures that compose the limbic system, this region seems to have the ability to interpret the intentions of others (and is possibly correlated to systems of mirror neurons). Above AB20, in the mean temporal gyrus, is AB21, whose function is still uncertain, but it seems involve the ability to recognize faces (known physiognomy), to understand the meaning of words during reading, and to estimate distances. Along with AB22, AB21 assists in the sound comprehension of melodies. An integral part of the area of language or area of Wernicke, AB22 is located in the upper temporal gyrus, circling primary auditory area 41 and associative auditory area 42 , insinuating into the interior of the parietal lobe, marking its limit with Wernicke area 29. Some authors subdivide AB22 into 22A and 22B, and the latter, when injured, is associated with Wernicke aphasia or expression, when the interpretive capacity is severely impaired, without speech involvement. AB41 and $\mathbf{4 2}$, which are located in the upper temporal gyrus, are considered primary and associative auditory areas respectively. This region has a tonotopic map, with sound intensity (acute-severe) recognition. The lesions in the transverse temporal gyrus of Heschl (primary auditory area), bilaterally, are bilateral cortical deafness. Possibly, in this transverse temporal gyrus, there is a circuit of mirror neurons, which may be activated when the individual visualizes the orofacial gestures related to pronunciation. ${ }^{17,18}$

AB37, a region of discontinuity of the middle and inferior temporal gyrus, is located in the transition between the temporal and occipital lobes. Currently, there is a relative consensus on its functionality: colorful visual information; naming (with involvement of the memory circuits); and facial, numerical and lexicon recognition. Lesions in AB37 may course clinically with semantic paraphasia and difficulty in finding words during a conversation, anomy, prosopagnosia and visuo-constructive apraxia. ${ }^{19} \mathbf{A B 3 8}$ is in the anterior pole of the temporal lobe, and is related to complex emotional responses. In Alzheimer disease, this will be one of the first areas involved, usually presenting with epileptic seizures of the temporal lobe. Studies with fNMR revealed that this area participates in several neurocircuits, including: working memory, phonognosia (recognition of familiar sounds, aversive and pleasurable response to sounds), language processing, and executive and emotional functions. The olfactory pathways, unlike other sensory modalities, do not have a specific location in the cerebral cortex; several areas receive their afferences: the hypothalamus, the thalamus, the amygdaloidal complex, the prefrontal area, and AB34 (temporal lobe uncus). 
Lesions in AB34 are related to epileptic seizures of the temporal lobe that course with cacosmy. ${ }^{20,21}$ Anatomically, the parietal lobe is subdivided into the upper and lower parietal lobes, and the lower is formed by the angular gyrus and supramarginal gyrus. The angular rotation corresponds to $\mathbf{A B 3 9}$, that is, part of the Wernicke area, which is responsible for the concepts of quantification, and spatial orientation, that is, for mathematics. The lesions in this region of the left hemisphere develop with Gerstmann syndrome (digital agnosia, temporospatial disorientation, dysgraphia and developmental dyscalculia), a semantic aphasia. The angular rotation region has a great associative capacity: auditory, spatial and visual information, being one of the last regions to mature. Arithmetic, somatognosia and language (reading) skills are attributed to the angular gyrus. In particular, the angular rotation of the right hemisphere correlates with visuo-spatial processing, and its lesions are associated with heminegligence. In parallel, studies with fNMR showed that this region also acts in verbal creative processing. Laterally to the angular gyrus, composing the inferior parietal lobe, there is the supramarginal gyrus, or $\mathbf{A B 4 0}$, another Wernicke area component. This region has great activity in complex verbal processing, creativity and semantic processing. Clinically, lesions in this region may result in Wernicke aphasia or sensory transcortical aphasia. In the right hemisphere, the supramarginal gyrus region correlates with self-reflection. ${ }^{22,23}$ Still in the parietal lobe, but in the uttermost lateral portion of the postcentral gyrus, is AB43, which is considered the primary gustatory cortex. Lesions in this area still have an uncertain character and may course with dysgeusia or ageusia. The motor area of the speech, or Broca area, classified by Brodmann as AB44, is in the inferior frontal gyrus, in its pars opercularis and triangularis. Neuroimaging revealed that, in individuals diagnosed with autistic spectrum disorder, including Asperger syndrome, there is a significant reduction in gray matter in $\mathrm{AB} 44$. Some authors consider the Broca area as the association between AB44 and AB45. According to recent studies, the anterior insula should be included in the so-called Broca area due to its involvement in speech motor planning (phonological and semantic fluency). Clinically, lesions in these regions are called motor aphasia (or Broca aphasia), and its variant, transcortical motor aphasia. The working memory uses this region intensely in the production and search for the correct words to be used according to each occasion. It is also worth noting that the Broca area is responsible for speech itself in the left hemisphere, giving this hemisphere its dominant characteristic. Intonation, melody and prosody correlate to the functions of the Broca area of the non-dominant hemisphere, which, in general, is the right hemisphere. Still with motor characteristics, the system of mirror neurons seems present in this region by internally mimicking a movement only visualized and previously learned. In the most anterior portion of the frontal lobe, AB9 and 46 represent the dorsolateral prefrontal (DLPF) cortex. However, fNMMR exams reveal that this region corresponds to AB9, 10, 11, 12, 45, 46 and to the upper portion of $\mathbf{A B 4 7} .^{24,25}$ These areas are related to the integration between somatosensory information and motor plan- ning and response, including associated intellectual functions. The DLPF cortex represents working memory itself, and practically all intellectual functions that require cortical and subcortical participation use the DLPF. AB32, which is located in the prefrontal cortex, receives several fascicles (not only the arched fascicle, but also the longitudinal and uncinated fascicles) of the Wernicke area, possessing special importance in the intelligence circuit when choosing one of the various hypotheses of possible answers to a problem, thus forwarding its final information to the Broca area. Clinically, the lesions in the DLPF cortex result in executive dysfunctions and impairment of affection, social judgment, executive memory and abstract thinking. The lower and central portions of $\mathbf{A B 4 7}$ are related to semantic and phonological processing, besides assisting in decisionmaking (cost versus benefit). In - Table 1, the cerebral cortex is separated by lobes (frontal, parietal, temporal, occipital and limbic lobes), highlighting their main functions and clinical syndromes correlated with the corresponding $A B$, as well as their treatment. ${ }^{14,22-24,26-33}$

The Fick principle and cerebral metabolism: fMNR. Adolf Eugen Fick developed a technique to measure the cardiac output by establishing a physical law with which it becomes possible to measure the metabolic rate of an organ from the clearance of a serum marker. Seymour Kety and Carl F. Schmidt adapted the Fick principle to study cerebral metabolism. In 1990, Seiji Ogawa and David Tank presented the fNMR technique using the BOLD method to estimate cerebral metabolism through the brain's oxygen consumption from arterial blood. The fNMR detects changes in the content of deoxyhemoglobin within a unit of volume in the encephalon. Applying the Fick law, the concentration of deoxyhemoglobin is proportional to the cerebral metabolic rate for oxygen divided by the cerebral blood flow. It is, thus, through energetic metabolism that fNMR can reveal the cerebral metabolic rate in the various areas during a task. The fNMR, therefore, is not able to reveal the activity of a neuron, but reflects the metabolism of that cerebral region. The fNMR differs from PET, since, in the latter, there is a need for the administration of radionucleotides in the bloodstream that emit positrons (positive loads of a particle), behaving like the deoxyhemoglobin used by the fNMR. However, here, there are gamma wave emissions. Despite the great advances in neuroscience with the fNMR, there are limitations. There are four variables that correlate with brain metabolism: 1) glucose uptake; 2) cerebral blood flow; 3) cerebral blood volume; and 4) deoxyhemoglobin content, the latter being the basis of the BOLD technique. The content of deoxyhemoglobin cannot be measured in absolute terms, resulting from the correlation between cerebral blood flow, cerebral blood volume and the basal metabolism of the cortical region (or resting state). It is clear, therefore, that different cortical regions, with different metabolisms in resting states, will present different BOLD values, which may originate false functional interpretations. Another limiting factor is the use of BOLD fNMR techniques in patients with vasculopathies: neuroimaging alterations may be more correlated to perfusion deficit than to neuronal (functional) 
Table 1 Correlation of anatomo-clinical findings with Brodmann's areas, separated by lobes, with identification of their corresponding tracts

\begin{tabular}{|c|c|c|}
\hline \multicolumn{3}{|l|}{ FRONTAL LOBE } \\
\hline Clinical manifestation & Brodmann's areas & Correlated tracts \\
\hline \multicolumn{3}{|l|}{ 1. Motor syndrome } \\
\hline Hemiparesia, hemiplegia & 4,6 & Corticospinal tract \\
\hline Apraxia & 6,8 & ULF \\
\hline Abnormality of conjugated gaze & $8,9,46$ & MLF and cingulate \\
\hline \multicolumn{3}{|l|}{ 2. Cognitive syndrome } \\
\hline $\begin{array}{l}\text { Memory, abstract thinking and behavior } \\
\text { inhibition deficits }\end{array}$ & $8,9,47$ & $\begin{array}{l}\text { ULF, uncinate and frontostriatal (internal } \\
\text { capsule) }\end{array}$ \\
\hline $\begin{array}{l}\text { Dysexecutive symptoms } \\
\text { (learning, planning, focus) }\end{array}$ & $6,8,9$ & Frontostriatal (internal capsule) \\
\hline Aprosodia/Motor afasia (Broca) & $6,44,45$ & Arched fascicle \\
\hline Heminegligence & 6 & ULF \\
\hline Ideomotor apraxia & 6,8 & ULF, corpus callosum \\
\hline \multicolumn{3}{|l|}{ 3. Abulia } \\
\hline Loss of interest & $10,11,24,32,33$ & Subcallous cingulate \\
\hline Mutism and akinesia & $6,8,9,24,32$ & Dorsal cingulate and ULF \\
\hline Lack of concentration and loss of motivation & $8,9,10,24,32,33$ & Anterior cingulate and inner capsule \\
\hline \multicolumn{3}{|l|}{ 4. Behavioral syndrome } \\
\hline $\begin{array}{l}\text { Personality alterations, emotional lability, social } \\
\text { disinhibition, hypersexuality, motor imitation, } \\
\text { primitive reflexes (grasp/hold), impulsiveness }\end{array}$ & $10,11,47$ & $\begin{array}{l}\text { Uncinate, frontostriatal (internal capsule) e } \\
\text { LFOF }\end{array}$ \\
\hline Dysphemia & $6,44,45$ & Anterior thalamic projection \\
\hline \multicolumn{3}{|l|}{ PARIETAL LOBE } \\
\hline Clinical manifestation & Brodmann's areas & Correlated tracts \\
\hline \multicolumn{3}{|l|}{ 1. Somatosensory and tactile syndrome } \\
\hline Disestesia, hipoestesia, hyperestesia, alodinia & $3 a, 3 b, 1,2$ & Inner capsule (superior thalamic projection) \\
\hline Hallucination and tactile perseveration & $3 a, 3 b, 1,2,5,40$ & Inner capsule (superior thalamic projection) \\
\hline Stereognosis, agrafoestesia, extinction & $5,7,40$ & $\begin{array}{l}\text { ULF, inner capsule (superior thalamic } \\
\text { projection) }\end{array}$ \\
\hline \multicolumn{3}{|l|}{ 2. Motor syndrome } \\
\hline Ataxia & $5,7,19$ & ULF, cingulate \\
\hline Apraxia (ideomotor, ideational, constructive) & $5,7,39,40$ & ULF and arched, corpus callosum trunk \\
\hline Oculomotor apraxia & 5,7 & ULF \\
\hline \multicolumn{3}{|l|}{ 3. Language syndromes } \\
\hline $\begin{array}{l}\text { Dyscalculia, alexia, agraphia, conduction } \\
\text { aphasia, impairment of verbal memory, anomia, } \\
\text { dyslexia, Gerstmann syndrome }\end{array}$ & 39,40 & Arched fascicle \\
\hline \multicolumn{3}{|l|}{ 4. Complex visual syndromes } \\
\hline Unreality sensation, out-of-body experiences & 39,19 & Thalamic projection and arched volume \\
\hline \multicolumn{3}{|l|}{ OCCIPITAL LOBE } \\
\hline Clinical manifestation & Brodmann's areas & Correlated tracts \\
\hline \multicolumn{3}{|l|}{ 1. Simple visual syndromes } \\
\hline $\begin{array}{l}\text { Scotomas, phosphonae, quadrantanopsia, } \\
\text { hemianopsia, blindness }\end{array}$ & 17 (V1 area) & Optical radiation \\
\hline
\end{tabular}


Table 1 (Continued)

\begin{tabular}{|c|c|c|}
\hline \multicolumn{3}{|l|}{ FRONTAL LOBE } \\
\hline Clinical manifestation & Brodmann's areas & Correlated tracts \\
\hline \multicolumn{3}{|l|}{ 2. Dorsal visual syndromes } \\
\hline $\begin{array}{l}\text { Acinetopsia, visual hallucination of motion, } \\
\text { Alloesthesia }\end{array}$ & 19 (V3 area) & ULF \\
\hline \multicolumn{3}{|l|}{ 3. Ventral visual syndromes } \\
\hline $\begin{array}{l}\text { Achromatopsia, visual agnosia, alexia, visual } \\
\text { hallucination, prosopagnosia, visual amnesia, } \\
\text { Capgras syndrome, Fregoli syndrome }\end{array}$ & 18 (V2 area), 19, 37 & LLV, lower fronto-occipital fascicle \\
\hline \multicolumn{3}{|l|}{ TEMPORAL LOBE } \\
\hline Clinical manifestation & Brodmann's areas & Correlated tracts \\
\hline \multicolumn{3}{|l|}{ 1. Hearing circuit } \\
\hline Cortical deafness, dysacusis & 41 & Acoustic radiation \\
\hline \multicolumn{3}{|l|}{ 2. Language syndromes } \\
\hline $\begin{array}{l}\text { Wernicke aphasia, transcortical sensory aphasia, } \\
\text { conduction aphasia, anomia }\end{array}$ & $42,22,37$ & \\
\hline \multicolumn{3}{|l|}{ 3. Behavioral syndromes and memory } \\
\hline Semantic dementia & $20,21,38$ & Uncinated fascicle and LLV \\
\hline Reduction of verbal fluency & 38 & Uncinated fascicle \\
\hline \multicolumn{3}{|l|}{ LIMBIC LOBE } \\
\hline Clinical manifestation & Brodmann's areas & Correlated tracts \\
\hline \multicolumn{3}{|l|}{ 1. Hypothalamic-hippocampal syndrome } \\
\hline Anterograde and retrograde amnesia & 28 & Fornix and mammillothalamic tract \\
\hline Alzheimee (early stage) & $23,26,28,29,30,35$ & Fornix and posterior cingulate \\
\hline Korsakoff & & Mammillothalamic tract \\
\hline Topographic disorientation & $26,29,30,35$ & Cingulate, LLV \\
\hline \multicolumn{3}{|l|}{ 2. Amygdalo-orbitofrontal syndrome } \\
\hline $\begin{array}{l}\text { Klüver-Bucy syndrome (apathy, hyperorality, } \\
\text { perversion of appetite, visual agnosia, } \\
\text { hypersexuality) }\end{array}$ & $28,34,36,38$ & Uncinate, LLV \\
\hline Personality changes & $11,32,33,34$ & Uncinate e cingulate \\
\hline \multicolumn{3}{|l|}{ 3. Paralimbic syndromes } \\
\hline Indifference to pain & $24,32,33$ & Anterior cingulate and thalamic projection \\
\hline $\begin{array}{l}\text { Hyposmia (common in Parkinson and } \\
\text { Alzheimer), hyperosmia }\end{array}$ & $12,25,32$ & Cingulate \\
\hline Apathy & $24,32,33$ & Anterior cingulate \\
\hline Depression, irritability & $24,25,32,33$ & Subcallosum cingulate \\
\hline Semantic dementia & 36,38 & Uncinate, LLV \\
\hline
\end{tabular}

Abbreviations: LFOF, lower fronto-occipital fascicle; LLV, lower longitudinal volume; MLF, medial longitudinal fascicle; ULF, upper longitudinal fascicle. Note: modified from Catani et al, 2012. ${ }^{33}$

deficit itself. The inhibitory synapses also heal with higher metabolic demand, increasing the BOLD signal with the inhibitory character of that cortical region. And, finally, the experiments with the BOLD technique used in the same individual are irreproducible with time, since, with learning, neurons naturally consume less energy to perform the same function due to their neuroplasticity. 1,32,34-36

New proposed subdivisions. After Brodmann's publication in $1909,{ }^{30}$ a new cortical functional map is published for the first time ( $\mathbf{F i g . ~ 2 ) . ~ S i n c e ~ B r o d m a n n , ~ n e u r o s c i e n t i s t s ~ t r y ~}$ to subdivide the cortical and subcortical areas to update them with the new knowledge obtained by neuroimages. Subareas differ microstructurally, presenting a functional specialization, forming a real connection network, which has been identified, at least partially, nowadays. Glasser et $\mathrm{al}^{2-5}$ published, in 2016, the most recent cortical subdivision since 1909. According to the authors, each hemisphere has $\sim 50$ to 200 functional areas, based on its topography, 


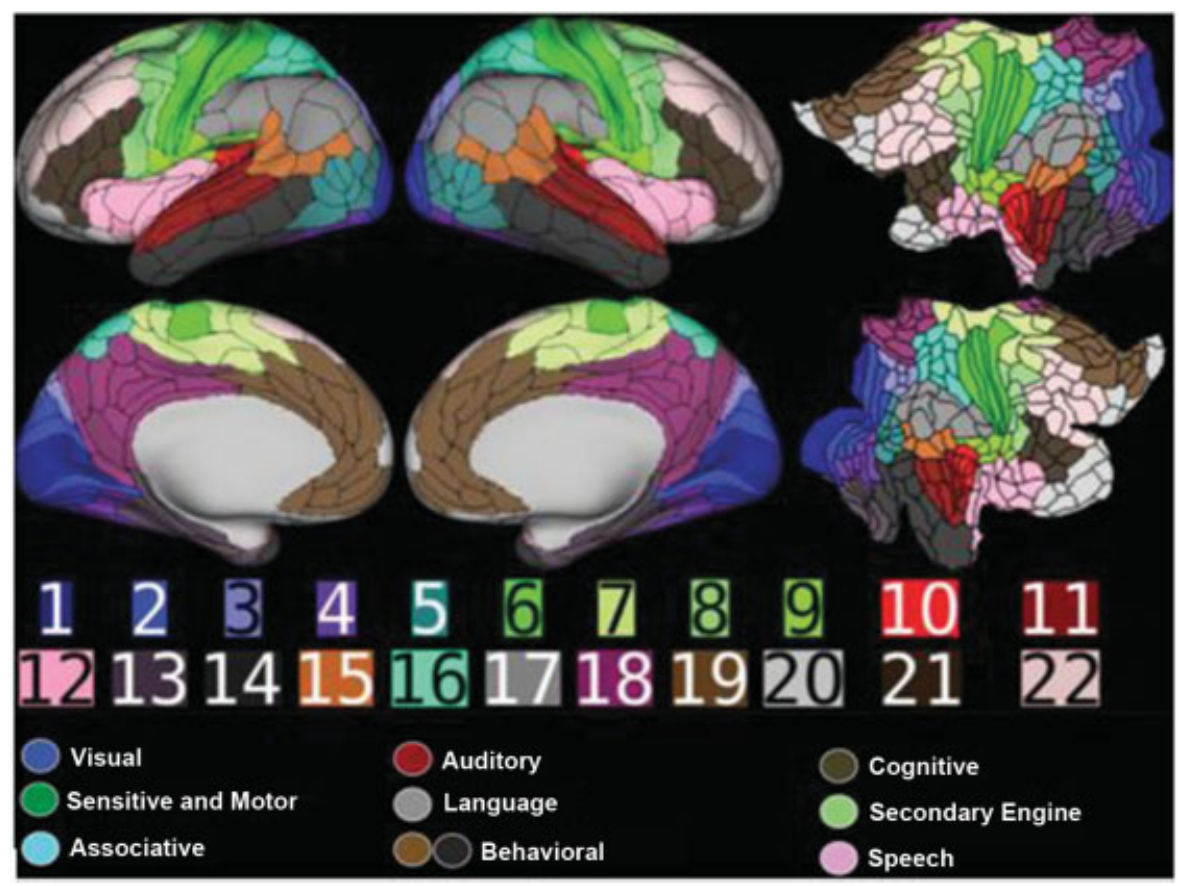

Fig. 2 New cerebral cortical functional map with 180 areas present in each hemisphere and some regional functional characteristics. The numbers represent the 22 large regions separated by Glasser et al: ${ }^{2-5}$ (1) primary visual cortex (V1); (2) visual cortex (V2/V3); (3) dorsal visual; (4) ventral visual; (5) MT complex; (6) sensorimotor cortex; (7) cingulate rotation and sensorimotor paracentral lobe; (8) premotor cortex; (9) posterior opercular cortex; (10) primary auditory cortex; (11) secondary auditory cortex; (12) frontal and insular opercular cortices; (13) medial temporal cortex; (14) lateral temporal cortex; (15) sensory area: parieto-occipitotemporal junction; (16) upper parietal; (17) inferior parietal; (18) posterior cingulate; (19) medial anterior and pre-frontal cingulate; (20) frontal orbitopolar; (21) lower frontal; (22) dorsolateral prefrontal. Source: Adapted from Glasser et al (2016). ${ }^{2-5}$

interconnection, cytoarchitecture and function. Using the fNMR data from the Human Connectome Project (HCP) database, the degree of cortical myelinization and cortical thickness were observed by T1- and T2-weighted MRI images, while cortical function was verified by BOLD fNMR during multitasking testing. The connectivity and topography were also evaluated by fNMR in the cerebral resting states. Glasser et $\mathrm{al}^{2-5}$ described 180 functional areas present in each cerebral hemisphere, all confirmed by the methodology adopted by the authors. Of the 180 areas, 83 were named according to their function previously described by numerous neuroanatomical scientific studies that used invasive methods, others were simply subdivided (such as BA 31, BA 31a [Anterior Brodmann Area 31], 31PD [Posterodorsal BA 31], 31PV [Posteroventral BA 31], for example), and some areas received new names. The similarity between the functional areas of both hemispheres is impressive. The data published by Glasser et $\mathrm{al}^{2}$ have important clinical implications for neurosurgical interventions. Through noninvasive methods of neuroimaging, these professionals can obtain extremely detailed functional maps of their patients, thus reducing surgical morbidity. Compared with inferior animals, the human cerebral cortex becomes much more complex because of the cognitive functions present in the dorsolateral, temporal and parietal prefrontal regions.

The authors subdivided the 180 areas into 22 functional regions. The first group consists of five regions involved with visual function (essentially, the occipital lobe): 1) the primary visual cortex (including area V1 [AB17]); 2) the visual cortex (areas V2 and V3 [AB18 and 19, and also the AB19 subdivided into hOC3v [ventral third visual area -V3], hOC3d [dorsal third visual área - V3], hOC4v [ventral fourth visual area], hOC4d [dorsal fourth visual area], hOC4lp [lateroposterior fourth visual area - V4], hOC4la [lateroanterior fourth visual area V4], hOC5v [ventral fifth visual area], hOC5d [dorsal fifth visual area]); 3) the dorsal visual (V3A, V3B, V6, V6A, V7 and IPS1 [Intraparietal sulcus area 1]), responsible for the spatial location of the visualized object; 4) the ventral visual (V8, the ventral visual complex [VVC], the PIT (Posterior Inferotemporal) complex, the fusiform facial complex [FFC], the ventromedial visual area [VMV1, 2 and 3]), which is responsible for recognition of visualized objects and facial recognition; 5) and the MT (Middle Temporal Area) complex and neighboring areas, which are formed by the side of the occipital lobe and the posterior temporal cortex, which are cell groups that perceive moving images, adjusting the conjugated movement of the gaze via the intraparietal sulcus and AB8. ${ }^{2-5,37}$

The second group consists of four areas with primary sensory and motor functions; Therefore, the frontal and parietal lobes, essentially: 6) the sensorimotor cortex (AB3a, b, 1, 2 and 4, which are surrounded by the 6MP (Supplementar Motor Area 6), 6d, frontal eye field [FEF], 55b and 6v areas); 7) the rotating medial 24dd (cingulate motor cortex dorsal area 24), 24dv (cingulate motor cortex ventrodorsal area 24) gyrus and sensorimotor paracentral lobe $6 \mathrm{ma}$ (Anterior motor área 6), SCEF (supplementary and cingulate eye field), $5 \mathrm{~m}$ (motor area 5), 5L (lateral motor area 5), 5MV (ventral motor area 5); 8 ) the premotor cortex (55b, 6d, 6a, 6v, 6r; AB8 was subdivided 
into the FEF and prefrontal eye field [PEF]); and 9) the posterior opercular cortex SII (BA 5 and BA 7).

The third group consists of three functional regions related to hearing, essentially corresponding to the temporal lobe: 10) the primary auditory cortex (AB41 replaced by A1 and several subdivisions: (medial belt), LBelt (lateral belt), PBelt (posterior belt), RI (retro-insular cortex)); 11) the secondary auditory cortex (AB42 subdivided into 8 areas); 12) the frontal and insular opercular cortices (which are subdivided into 13 areas: area 52, the parainsular [PI] cortex, the granular index [GI], the posterior insula [PoI1 and Pol2], the frontal opercular area [FOP2 and FOP3], the middle insular $[\mathrm{MI}]$ area, the anteroventral insular $[\mathrm{AVI}]$ area, the complex of the anterior agranular insular [CAAI] area, the piriformis [PIR] cortex, FOP4 and FOP5).

The fourth group consists of two areas that complement the functions related to the temporal lobe: 13) the medial temporal cortex (hippocampus, presubiculum, entorhinal cortex, perientorhinal cortex, ectorhinal complex and peri-hippocampal areas 1,2 and 3 ); 14) and the lateral temporal cortex (which is subdivided into nine areas). The fifth group consists of a large area composed of four functional regions present in the posterior parietal lobe: 15) the sensory area: the parietooccipitotemporal junction (AB39 and 40, which are subdivided into 5 multimodal areas: TPOJ1 (temporo-parieto-occipital junction 1), ТРОJ2 (temporo-parieto-occipital junction 2), TPOJ3 (temporo-parieto-occipital junction 3), the upper visual temporal [UVT] area, and the perissilvian language [PSL] area), formed by the tertiary associative areas or Wernicke area, which is responsible for language; 16) the upper parietal areas (areas around the intraparietal sulcus, AB5 and 7: LIPv (ventral lateral intraparietal area), LIPd (dorsal lateral intraparietal area), VIP (ventral intraparietal complex), AIP (anterior intraparietal complex), MIP (medial intraparietal area), 7am (anteromedial BA 7), 7pl (posterolateral BA 7), 7al (anterolateral BA 7), $7 \mathrm{pm}$ (posteromedial BA 7)), which are subdivided into ten associative areas between the visual and somatosensory functions; 17$)$ the inferior parietal area (subdivided into ten areas: IP0 (intraparietal area 0), IP1 (intraparietal area 1), IP2 (intraparietal area 2), PFm (prefrontal motor complex), PF (prefrontal complex), PFop (opercular prefrontal area)), an area that suffered significant expansion in the human brain when compared with other animals, and is part of the language area (classically, AB39 and 40); 18) the posterior cingulate (subdivided into 14 areas), which participates in several associative neurocircuits, highlighting the dorsal visual transitional (DVT) cortex, the extrastriated visual cortex, the parieto-occipital sulcus (POS1 and POS2) region and the retrosplenic complex (AB29 and 30).

The last four functional anterior areas cover the frontal lobe: 19) the anterior and medial prefrontal cingulates (classically described as AB32, 33 and 24, they are subdivided into 15 areas, with emphasis on $\mathrm{AB} 32$, or the paralimbic cortex, which is divided into D32 (dorsal BA 32), P32 (posterior BA 32), S32 (superior BA 32), P32PR (posterior prime segment of BA 32), A32PR (anterior prime segment of BA $32)$ ), which are formed by a region interconnected to several circuits, including cognitive areas, primary motor areas and premotor areas; 20 ) the orbitopolar frontal cortex (which is subdivided into 11 areas and complexes); 21) the inferior frontal cortex (including AB44 and 45 or drill area, which is subdivided into 44d (dorsal BA 44), 44v (ventral BA 44), 45a (anterior BA 45), 45p (posterior BA 45), 471 (lateral BA 47)); 22) the DLPF, which is formed by the largest functional heterogeneous area present in the human neocortex; this area has similarities with the inferior parietal lobe and lateral temporal cortex, also forming one of the tertiary associative areas. It is subdivided into 13 areas: 9A (anterior BA 9), 9P (posterior BA 9), 46 (BA 46), i6-8 (interior transitional BA 68), s6-8 (superior transitional BA 6-8) 8AD (anterodorsal BA 8), P9-46v (posterior transitional BA 9-46), A9-46v (anterior transitional BA 9-46), 9-46d (dorsal transitional BA 9-46). There is a remarkable interhemispheric asymmetry in the SFL region (upper frontal language), which is larger on the left, and is involved in the language circuit. ${ }^{2-5,38-40}$

Genetics and the fNMR. An additional step to the macroscopic functional mapping would be to add the gene pattern to the studied areas, correlating them with their transcriptional profiles. For that, Peng et al ${ }^{6}$ separated the cerebral cortex into 12 functional areas, determined by fNMR, and studied their gene expression patterns, comparing them in more than two thousand individuals. A huge similarity was found between the gene expression of these fNMR preselected areas, showing that the functional macroscopic division proposed was feasible. With these genetic data, it becomes possible to identify a functional brain region only by the pattern of expression of some genes. Additionally, the authors found several gene coexpressions ( $71.4 \%$ of the genes) belonging to the 4 studied lobes (frontal, parietal, temporal and occipital lobes), attributing to the frontal lobe the greatest presence of specific genes, which is not found in the other lobes. ${ }^{6,41-44}$

\section{Conclusion}

Korbinian Brodmann revolutionized neuroscience by publishing, in 1909, his functional map of the human cerebral cortex. More than creating a map, Brodmann paved the way for the understanding of diseases, behaviors and the normal functioning of the human brain. Based on his concepts, new works were performed and added information, filling a huge functional gap that was still present. Only in the decades of 1980 and 1990, when the neuroimaging exams began to reveal more concrete data on the functioning of a living brain, it was possible to correlate neuroanatomy with neurophysiology in vivo. The technology continues to advance both in the macroscopic and microscopic aspects, offering a unique window of opportunity for a cerebral remapping, or rather an increment to the map drawn in 1909 . Glasser et al ${ }^{2-5}$ published, in 2016, the first map since 1909, presenting 180 functional areas in each cerebral hemisphere, based on topographic regions, myelinization patterns and cortical thickness of the different regions, BOLD fNMR techniques with responses to multitasking in vivo and brain functioning in their resting state, offering neuroscience a unique moment of interpretation of the cerebral functions. It is worth noting that, even with this technological arsenal, it is still very 
difficult to determine a common pattern, because each individual is unique and has neuro-anatomo-functional particularities, leaving the neuroscientists, neurologists and neurosurgeons to explore them in an individualized way.

\section{Conflict of Interests}

The authors have no conflict of interests to declare.

\section{References}

1 Raichle ME. A brief history of human brain mapping. Trends Neurosci 2009;32(02):118-126

2 Glasser MF, Coalson TS, Robinson EÇ, et al. A multi-modal parcellation of human cerebral cortex. Nature 2016;536(7615):171-178

3 Glasser MF, Coalson TS, Robinson EÇ, et al. Supplementary Neuroanatomical Results For A multi-modal parcellation of human cerebral cortex. Nature 2016

4 Glasser MF, Coalson TS, Robinson EÇ, et al. Supplementary Methods For A multi-modal parcellation of human cerebral cortex. Nature 2016

5 Glasser MF, Coalson TS, Robinson EÇ, et al. Supplementary Results and Discussion For A multi-modal parcellation of human cerebral cortex. Nature 2016

6 Peng $\mathrm{Q}$ Schork A, Bartsch $\mathrm{H}$, et al; Pediatric Imaging, Neurocognition and Genetics Study; Alzheimer's Disease Neuroimaging Initiative. Conservation of Distinct Genetically-Mediated Human Cortical Pattern. PLoS Genet 2016;12(07):e1006143

7 Zilles K, Amunts K. Centenary of Brodmann's map-conception and fate. Nat Rev Neurosci 2010;11(02):139-145

8 Brodmann K. in Allgemeine Chirurgie der Gehirnkrankheiten, 86426 (Verlag von Ferdinand Enke, Stuttgart, 1914).

9 Toga AW, Thompson PM, Mori S, Amunts K, Zilles K. Towards multimodal atlases of the human brain. Nat Rev Neurosci 2006;7 (12):952-966

10 Zilles K, Amunts K. Receptor mapping: architecture of the human cerebral cortex. Curr Opin Neurol 2009;22(04):331-339

11 Johansen-Berg H, Rushworth MF. Using diffusion imaging to study human connectional anatomy. Annu Rev Neurosci 2009;32:75-94

12 Schweizer R, Voit D, Frahm J. Finger representations in human primary somatosensory cortex as revealed by high-resolution functional MRI of tactile stimulation. Neuroimage 2008;42(01):28-35

13 Schweisfurth MA, Frahm J, Schweizer R. Individual fMRI maps of all phalanges and digit bases of all fingers in human primary somatosensory cortex. Front Hum Neurosci 2014;8:658

14 Caspers J, Zilles K, Eickhoff SB, Schleicher A, Mohlberg H, Amunts K. Cytoarchitectonical analysis and probabilistic mapping of two extrastriate areas of the human posterior fusiform gyrus. Brain Struct Funct 2013;218(02):511-526

15 Kujovic M, Zilles K, Malikovic A, et al. Cytoarchitectonic mapping of the human dorsal extrastriate cortex. Brain Struct Funct 2013; 218(01):157-172

16 Klinge C, Eippert F, Röder B, Büchel C. Corticocortical connections mediate primary visual cortex responses to auditory stimulation in the blind. J Neurosci 2010;30(38):12798-12805

17 Morosan P, Rademacher J, Schleicher A, Amunts K, Schormann T, Zilles K. Human primary auditory cortex: cytoarchitectonic subdivisions and mapping into a spatial reference system. Neuroimage 2001;13(04):684-701

18 Thaut MH, Trimarchi PD, Parsons LM. Human brain basis of musical rhythm perception: common and distinct neural substrates for meter, tempo, and pattern. Brain Sci 2014;4(02):428-452

19 Ardila A, Bernal B, Rosselli M. Language and visual perception associations: meta-analytic connectivity modeling of Brodmann area 37. Behav Neurol 2015;2015:565871

20 Mori K, Takahashi YK, Igarashi KM, Yamaguchi M. Maps of odorant molecular features in the Mammalian olfactory bulb. Physiol Rev 2006;86(02):409-433
21 Neville KR, Haberly LB. The olfatory cortex. In: Shephered GM (ed). The synaptic organization of the brain. 2004:415-454New York: Oxford Univ. Press

22 Jung RE, Haier RJ. The Parieto-Frontal Integration Theory (P-FIT) of intelligence: converging neuroimaging evidence. Behav Brain Sci 2007;30(02):135-154, discussion 154-187

23 Dick AS, Tremblay P. Beyond the arcuate fasciculus: consensus and controversy in the connectional anatomy of language. Brain 2012; 135(Pt 12):3529-3550

24 Schneiderman JS, Hazlett EA, Chu KW, et al. Brodmann area analysis of white matter anisotropy and age in schizophrenia. Schizophr Res 2011;130(1-3):57-67

25 Zou N, Chetelat G, Baydogan MG, et al. Metabolic connectivity as index of verbal working memory. J Cereb Blood Flow Metab 2015; 35(07):1122-1126

26 Shah KB, Hayman LA, Chavali LS, et al. Glial tumors in brodmann area 6: spread pattern and relationships to motor areas. Radiographics 2015;35(03):793-803

27 Deary IJ, Penke L, Johnson W. The neuroscience of human intelligence differences. Nat Rev Neurosci 2010;11(03):201-211

28 Turkeltaub PE, Gareau L, Flowers DL, Zeffiro TA, Eden GF. Development of neural mechanisms for reading. Nat Neurosci 2003;6 (07):767-773

29 Colom R, Karama S, Jung RE, Haier RJ. Human intelligence and brain networks. Dialogues Clin Neurosci 2010;12(04):489-501

30 Brodmann K. Vergleichende Lokalisationslehre der Großhirnrinde in ihren Prinzipien dargestellt auf Grund des Zellenbaues (Barth, Leipzig, 1909); English translation available in Garey LJ. Brodmann's Localization in the Cerebral Cortex. (Smith Gordon. London, 1994).

31 Uhlhaas PJ, Singer W. Neural synchrony in brain disorders: relevance for cognitive dysfunctions and pathophysiology. Neuron 2006;52(01):155-168

32 Glasser MF, Van Essen DC. Mapping human cortical areas in vivo based on myelin content as revealed by T1- and T2-weighted MRI. J Neurosci 2011;31(32):11597-11616

33 Catani M, Dell'acqua F, Bizzi A, et al. Beyond cortical localization in clinico-anatomical correlation. Cortex 2012;48(10):1262-1287

34 Zhang D, Raichle ME. Disease and the brain's dark energy. Nat Rev Neurol 2010;6(01):15-28

35 Smith SM, Beckmann CF, Andersson J, et al; WU-Minn HCP Consortium. Resting-state fMRI in the human connectome project. Neuroimage 2013;80:144-168

36 Di X, Biswal BB; Alzheimer's Disease Neuroimaging Initiative. Metabolic brain covariant networks as revealed by FDG-PET with reference to resting-state fMRI networks. Brain Connect 2012;2(05):275-283

37 Buckner RL, Krienen FM. The evolution of distributed association networks in the human brain. Trends Cogn Sci 2013;17(12):648-665

38 Barch DM, Burgess GÇ, Harms MP, et al; WU-Minn HCP Consortium. Function in the human connectome: task-fMRI and individual differences in behavior. Neuroimage 2013;80:169-189

39 Tavor I, Parker Jones O, Mars RB, Smith SM, Behrens TE, Jbabdi S. Task-free MRI predicts individual differences in brain activity during task performance. Science 2016;352(6282):216-220

40 Van Essen DÇ, Glasser MF, Dierker DL, Harwell J, Coalson T. Parcellations and hemispheric asymmetries of human cerebral cortex analyzed on surface-based atlases. Cereb Cortex 2012;22(10):2241-2262

41 Strike LT, Couvy-Duchesne B, Hansell NK, Cuellar-Partida G, Medland SE, Wright MJ. Genetics and brain morphology. Neuropsychol Rev 2015;25(01):63-96

42 Chen C-H, Gutierrez ED, Thompson W, et al. Hierarchical genetic organization of human cortical surface area. Science 2012;335 (6076):1634-1636

43 Hawrylycz MJ, Lein ES, Guillozet-Bongaarts AL, et al. An anatomically comprehensive atlas of the adult human brain transcriptome. Nature 2012;489(7416):391-399

44 Gläscher J, Rudrauf D, Colom R, et al. Distributed neural system for general intelligence revealed by lesion mapping. Proc Natl Acad Sci U S A 2010;107(10):4705-4709 